MATEC Web of Conferences 22,03025 (2015)

DOI: $10.1051 /$ matec conf/20152203025

(C) Owned by the authors, published by EDP Sciences, 2015

\title{
Literature Review of Research on the Technology of Wire Rope Nonde- structive Inspection in China and Abroad
}

\author{
Jie Tian, Junying Zhou*, Hongyao Wang \& Guoying Meng \\ School of Mechanical Electronic and Information Engineering, China University of Mining and Technology, \\ Beijing, China
}

\begin{abstract}
As consumables in mine, wire ropes have great significance for safe operation of coal mines. The complex structure makes the nondestructive testing particularly difficult. This paper summarizes the existing methods of analysis at home and abroad from the perspective of strong magnetic and weakly magnetic; introduces the main methods of wire rope at the present, including principle and current status. At last, several critical problems in nondestructive testing of wire rope are discussed.
\end{abstract}

Keywords: wire rope; nondestructive testing technology; electromagnetic detection method

\section{INTRODUCTION}

As one of the most important flexible members, wire rope has high tensile strength and fatigue resistance, light weight, good elasticity, stable and reliable under dynamic load and overload ability and many other advantages. It is widely used in mine, metallurgy, ports, construction and other major industries of national economy and the improvement of department, transport and traction equipment. Wire ropes are widely used in in some areas such as cranes, oil drilling rig, elevators, and mine hoist. As a bearing component, wire rope will appear fatigue, corrosion, and even a sudden break because of various reasons. Its bearing capacity and reliability have the direct bearing on the safety of equipment and people. Because of the complicated structure of wire rope, the diversity of the work environment, the limitations of detection methods and the development of manufacturing technique, which make the defect detection of wire rope difficulty. It is very important to develop the nondestructive testing equipment which can detect the status of wire rope reliably. The nondestructive inspection technology of wire rope will be gradually developed.

There should be an appropriate detection method to conduct the nondestructive testing of wire rope. At present, there are many methods for nondestructive testing of wire rope including electromagnetic detection, ultrasonic detection method, X-ray detection, acoustic emission detection method, eddy current testing method and so on. The electromagnetic detection method has been widely used and promoted. The nondestructive detection method of wire rope will be analyzed and the status of the various detection methods will be introduced in the following parts.

*Corresponding author: zhoujunying716@163.com

\section{ANALYSIS OF THE PRESENT NONDE- STRUCTIVE DETECTION METHOD OF WIRE ROPE}

According to the nature and characteristics of the damage of wire rope, the damage of wire rope can be divided into Localized Flaw and Loss of Metallic Area [1]. The damage of LF refers to the wire rope damage which is locally produced, including the broken of internal and external, corrosion and local deformation. The characteristics of LF are that metallic cross-sectional area of wire rope which is suddenly decreased. The damage of LMA refers to the effective metallic sectional area of wire rope decrease slowly in an axial longer range, including wear, long distance of corrosion, and attenuation of wire diameter. The characteristics of LMA are that the metallic sectional area of wire rope which is slowly decreased in a longer range. Corresponding to these two kinds of damage, the traditional detection methods can be divided into LF magnetic flux leakage method [2], LMA/LF main flux method and LMA/LF loop flux method. The acoustic emission ultrasonic detection method which is lately claimed has been widely used.

\subsection{Inspection technology based on strong mag- netism}

The wire rope with good permeability was evenly magnetized along the axial direction. If the magnetization section of wire rope has a loss, it can appear in the magnetic leakage which causes the change of magnetic flux in magnetic circuit. Through the measurement of magnetic flux surface leakage of wire rope, it can detect the local damage such as broken wire and pitting. Through the measurement of main magnetic flux in magnetic circuit of wire rope, the damage of cross-sectional area and broken wire can be qualitatively detected. According to the different of detecting 


\section{MATEC Web of Conferences}

element, strong magnetic detection can be divided into the following kinds.

\subsubsection{Detection method based on Hall Sensor}

The Hall sensor detective method is a way which uses the Hall sensor as a sensor to detect flux changes of wire rope. Hall sensor is the semiconductor to which Hall effects are applied. The structure between the Hall sensor and the coil sensor are similar. But the former detective device is the Hall sensor which is set on the inner wall of the sensor. The latter is the induction coil which is around the wire rope. The detection principle of Hall sensor is that it placed near the surface of leakage magnetic field on the surface of wire rope and output the corresponding voltage signal. Although eliminating the impact which velocity has on signal amplitude, it still needs to transfer the signal of time domain to the spatial domain. The signal on the timeline will be stretched or compressed along with sampling speed, because the detective speed is not constant.

Hall sensor detective method being researched and applied abroad includes: From 1960s to 1990s, Kitzinger F. and Wint G.A. put forward an idea of using the Hall sensor as detecting device. The method tests the cross-sectional and partial area of wire rope and confirm the damage if it exists. Zawada Company has a Hall sensor which can accurately detect all serious defects including its position and depth. Local damage detection accuracy is $0.2 \%$ for the cross-sectional area of the wire rope [3]. Germany University of Stuttgart have designed a kind of sensor probe which is set the lining circular array with Hall sensors. It can clearly describe the wire rope of the axial and circumferential magnetic field [4]

Hall sensor detective method being researched and applied in the domestic includes: Yang Shuzi, Kang Yihua and other professors that come from Huazhong University of Science and Technology began to study the broken wire rope detection technology since 1980s. They used rare earth permanent magnet as excitation device which could activate wire rope. This method measured leakage magnetic field around the wire rope with the integrated Hall sensors. Through MTC-94 type of wire rope flaw detector and GDJY series of quantitative broken detector of wire rope, it achieves the quantitative detection of broken wire rope [5]. Tao Dexin of Wuhan University of Science and Technology uses the testing method of magnetic flux leakage by four Hall sensors to get the magnetic flux leakage signals and detect the wire rope damage qualitatively. Tan Jiwen et al in Qingdao University of Science and Technology design the steel wire rope exciter according to the principle of magnetic bridge circuit. They test the cross-sectional area of wire rope damage by Hall sensors [6]. Hunan Institute of Science and Technology applied for invention of detection device for broken wires, Hall sensors detect larger magnetic flux leakage after detection making alarm circuit light and buzzer [7].

\subsubsection{Detection method based on fluxgate sensor} Fluxgate detection is to obtain the testing signal by detecting the magnetic leakage field of wire rope defection. First of all, make the fracture defect produces the leakage magnetic field by using AC magnetic field for excitation. After that, the voltage in the coil will produce the symmetry changes, which can obtain the testing signal.

As shown in Figure 1, establish a loop by setting up single core and single coil sensor uniformly around the wire rope. Sensor signals perform badly by reverse tandem. Use AC magnetic field for excitation to remove the fixed wave signal brought by the wire rope. Therefore, it can increase the signal to noise ratio and restrain the mold interference in the detection of wire rope effectively. The advantages of fluxgate detection method are the high sensitivity and direction. The disadvantages are the complicated calculation, the enormous $\mathrm{AC}$ excitation equipment and difficult installation.
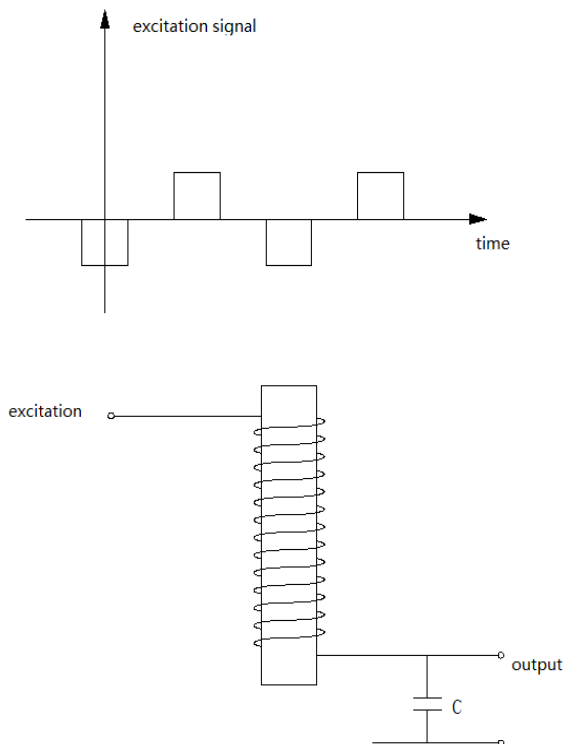

Figure 1. Fluxgate Assay

Canada Rotesco's Rotescograph 2D and 2C-TAG88M use fluxgate sensor [8]. It has higher detection accuracy to the local damage and the cross sectional area of the damage. The minimum length of the cross-sectional area damage can be resolved for 250 530mm. Gu Weixin from Shanghai Maritime University detects wire ropes by magnetic flux gate. Magnetic flux leakage signals of the defects on the surface of wire rope will be collected by the non-destructive detection probe based on single core and winding flux gate [9]. It has established the mathematical model [10] of wire rope testing signal and deduced the correlation function between the measured magnetic fields and the output voltage while 
adopting fluxgate detection of leakage magnetic field.

\subsubsection{Detection method based on induction coil}

The induction coil detection method is using permanent magnet to make the wire rope measured in deep saturation along the axial direction of the wire rope. Measure the damage of sectional area by measuring the axial flux of wire rope. Distinguish the local damage of wire rope by measuring the magnetic flux leakage of the surface of wire rope. The coil of induction coil detection method is gradually developed into a differential coil, and then split it into a differential coil. The biggest advantage of inductive coils is the low cost and easy to implement. The big drawback is that the speed of the rope detection greatly restricts the output signal of the sensor. Maintain high detection precision which is necessary to maintain the quantitative detection coil sensor speed. Instability detection speed will make the sensor signal changed. As shown from the pictures, Figure 2 is the original structure. Figure 3 is differential and Figure 4 is split differential coil. It emerged the first wire rope flaw which uses the AC induction coil sensor excitation in 1906. At present, the way of induction is developed from the AC excitation and DC excitation induction method into permanent DC excitation which is extensively used [2] Dr. H.R. Weischedel from American NDT conducted in-depth research. He made improvements to the detection coil and the coil detector circuit is added to the integrator circuit [11]. This detection device can quantitatively detect the damage of LMA, while the LF damage can be qualitatively analyzed. In China, Harbin Institute of Technology and a branch of Fushun Coal Mine using SCM system was successfully developed a GST-type wire rope flaw [12] which could detect LMA and LF simultaneously.

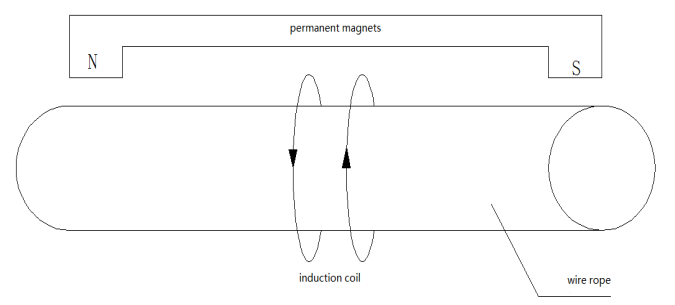

Figure 2. Original structure

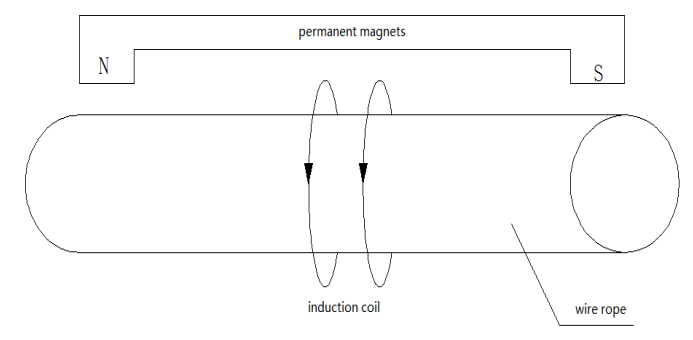

Figure 3. Differential coil

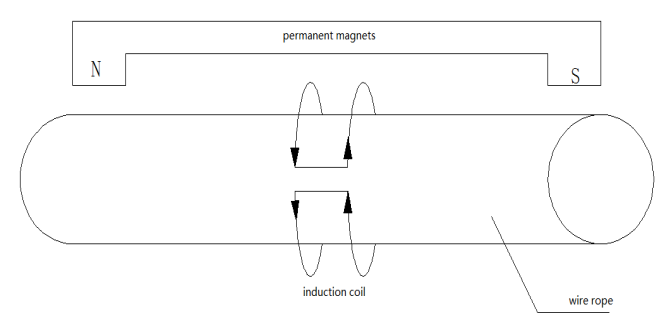

Figure 4. Split Differential Coil

\subsection{Inspection technology based on weak magnetic field}

The founding principle of weak magnetism inspection technology is the 'Spatial Magnetic Field Vector Resultants' theory. As an innovative and proven NDT, it adopts large air-space and non-contact weak magnetic testing devices. It is capable to detect quantitatively both internal and external flaws of the test wire rope by monitoring and extracting signals of magnetomotive force changes of the testing wire rope, which is preconditioned by weak magnetic loader. According to quantum mechanics, 'magnetic domains' exist among adjacent electrons of ferromagnetic medium, which are lined up in a certain shape because of the interactive and coupling effect of the electrons spin magnetic moment. In each magnetic domain, the direction of spin magnetic moment is same. W sensors are based on 'spatial magnetic field vector resultants' theory. The energy releasing device provides a given weak electromagnetic field $\mathrm{B}_{\mathrm{x}}$, which interacts with the weak magnetic field $B$ in each cross section of the test wire rope. They all generate the inductive magnetic field $\mathrm{B}_{\mathrm{y}}$, while the magnetism evaluating device converts the magnetometive force changes of $\mathrm{B}_{\mathrm{y}}$ into electric signals (See Figure 5).

Luoyang TCK Wire Rope Testing Technology Co., Ltd uses a new method to detect a variety of injuries quantitatively. Its TCK-BX can accurately evaluate the residual bearing capacity, the safety coefficient and the in-service life of wire rope.

\subsection{Acoustic emission-ultrasonic testing method [2]}

For the quantitative detection of the rope injury situation, in recent years, scholars at abroad also proposed a novel detection method that is acoustic emission ultrasonic testing method, which developed on the basis of the respective advantages of comprehensive ultrasonic acoustic emission (such as Figure 6). A set of transducers and evil mixture is introduced to the rope ultrasonic detection, modeling of acoustic emission signal characteristics of propagation in the rope; another set of transducers and evil mixture extract the signal, which is after amplification and sends the entry limit controller. And then calculate the stress wave coefficient to achieve quantitative identification rope defects. The testing method of acoustic emission - 


\section{MATEC Web of Conferences}

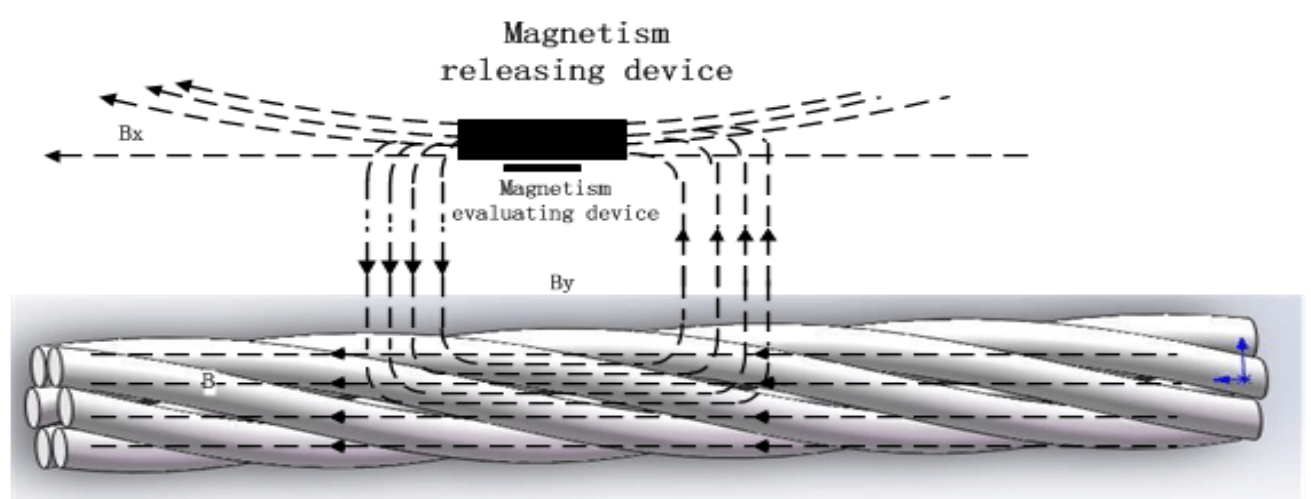

Figure 5. A Cross-sectional element

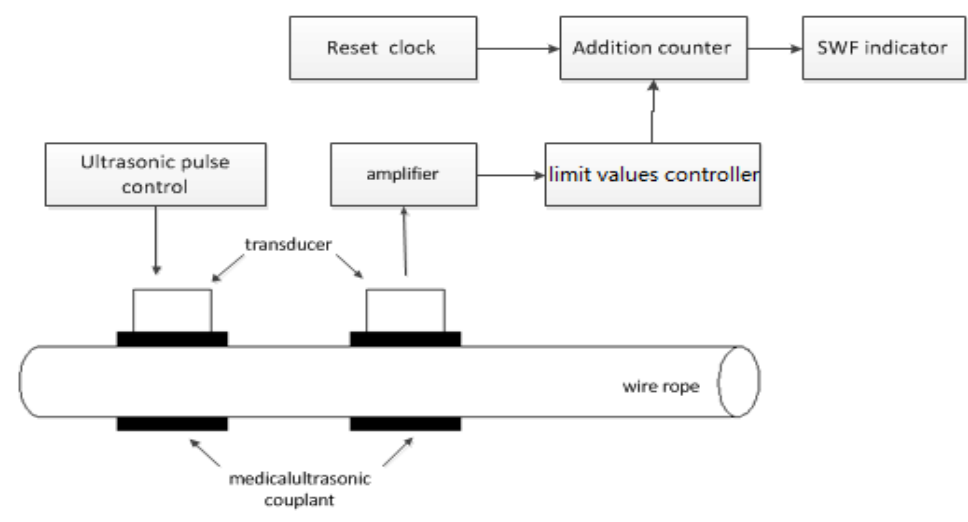

Figure 6. Acoustic emission-ultrasonic schematic

ultrasonic can not only carry out the rope defect detection and defect characterization, but also assess the fatigue damage of wire rope and internal performance including strength and hardness. This makes up the insignificancy of acoustic emission detection that only detecting stationary wire rope and capturing instantaneous signal of broken rope. Nevertheless, the method is still in the theoretical stage.

\section{$2.4 \quad$ X-ray detection method [13]}

When using ray to defect, different defects will have different fading. As shown in Figure 7, the ray intensity is $\mathrm{J}_{0}$ and the ray intensity which passes through the workpiece and defects are $\mathrm{J}_{\delta}$ and $\mathrm{J}_{\mathrm{X}}$. According to different ray intensity that film accepted, black poor parts are obviously reflected after washing. It can identify the defects of shape, position and so on.

Some domestic enterprises have a certain amount of research on X-ray detection. The x-ray detection device invented by Shanxi Hui Da uses different x-ray absorption rate having different density of material to make steel belt speed, high precision, full-face real-time scanned.

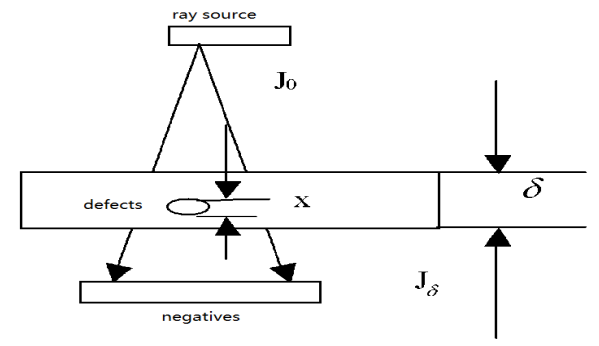

Figure 7. X-ray detection principle

\section{PROBLEMS OF NONDESTRUCTIVE TESTING OF WIRE ROPE TO BE SOLVED IN THE FU- TURE}

(1) Monitor the magnetic signal of fatigue damage of wire rope [14]. Using the magnetic detection technology to monitor the fatigue damage of ferromagnetic material is a new research direction on non-destructive testing techniques in recent years. Microstructure of ferromagnetic material is changed and it makes char- 
acteristics of magnetization changed, which causing fatigue damage. If the magnetic flux leakage detection technology is used to monitor the rope, the electromagnetic detection technology of wire rope will be lifted to a new level.

(2) The wire rope detection is easily disturbed. Using Hall element as a sensor, it will under electromagnetic interference. If it is near strong electromagnetic wave, the waveform will be detected interference. This appears larger fluctuation, which will affect the results of analysis. How to reduce or eliminate interference remains to be further studied.

(3) The relationship between the signal of LF and LMA and the strength loss of rope are uncertain. The purpose of detecting wire rope is to assess the damage of it. If we can build a mathematical model between LMA and residual strength with rope, then we will easily grasp the strength loss of rope. With the development of science and technology, it will become inevitable to develop intelligent equipment that can detect LMA and LF simultaneously.

(4) Reliability and usefulness of detection methods. Although there are some practical testing equipment at home and abroad, it can influence the results because of restrictions on principles and applications. So the reliability and correlation can't be satisfied to the application requirements of actual production site. Only as a secondary detection tool, its price and ease of use can be restricted.

\section{CONCLUSION}

So far, the main problems existing in technology and equipment of nondestructive testing of wire rope are low reliability, low intelligence, the lack of objectivity about test results and the incomplete information about the damage of wire rope. Thus we can't make fully estimations for the damage of wire rope. So we need to carry out exploratory research on the basis of the existing flaw detector and develop a new technology with high performance and reliability to meet the need of on-line detection. Through the deep analysis of various factors, we can quantify the scale of the influence of various factors on the grading and make grading standard for reference and contrast.

\section{ACKNOWLEDGMENTS}

The authors would like to thank the National Natural Science Foundation of China (No.51404276) and The Central Business Funds of Colleges (No.2014QJ01) for providing the financial support for conducting this research. The authors also thank the reviews for their useful comments and suggestions for improving the present manuscript.

\section{REFERENCES}

[1] Jia S M, 2002. Some views on the rope electromagnetic detection and strength assessment. NDT, 24(12): 522-525.

[2] Cao Y N, Zhang D L, Xu D G. 2005. Quantitative nondestructive testing of rope. NDT, 27(2): 91-95.

[3] Winter S, Briem U, Nussbaum J M. 1999. High resolution magnetic wire rope test-case study. OIPEEC Technical Meeting 'The Non-destructive Testing of Rope', Krakow: September, 1999. pp: 143-151.

[4] Jia S M, Ding K, Liu C W. 2000. MD-type wire rope flaw applications. Mining Machinery, 17(5): 15-18.

[5] Zhang D L, Cao Y N, Wang C, Xu D G. 2006. A new method of defects identification for wire rope based on three-dimensional magnetic flux leakage. Journal of Physics: Conference Series, 48: 334-338.

[6] Wang Y X, 2003. Development of MTC-B-type wire rope inspection rope testing device. Mining Machinery, (7): 4-7.

[7] Kang Y H, Yang K C, Yang S Z. 1992. Broken wire hall-effect magnetic flux leakage detection method based on structural characteristics of wire rope. Journal of Huazhong University of Technology, (S): 188-194.

[8] Ying L. 1998. Fluxgate mathematical model for wire rope NDT extremely signal acquisition. Journal of Shanghai Maritime University, 19(3): 39-44.

[9] Chai X L, Lu Y, Gan Z H, et al. 2009. Structure and character analysis of a new type of steel wire rope NDT detector apparatus. IEEE International Conference on Mechatronics and Automation, Changchun, pp: 4095-4099.

[10]Zhong X Y, Zhang X H. 2012. Optimal design of mine detection sensors. Coal Mine Machinery, 33(8): 7-9.

[11] Weichedel H R. 1987. Method and apparatus for magnetic inspection. United States Patent, 4: 659-991.

[12] Xu D G, Zhang X Y, Song D L. 1996. Intelligent portable wire rope flaw research. Instrument Technique and Sensor, (1): 22-25.

[13]Ruan W, Zheng M S. 2005. X-ray imaging system designed to detect rope. Instrumentation and Testing Technology, 24(12).

[14]Zhou Q. 2002. Research Rope Force Magnetic Effect of Fatigue Damage and Leakage Magnetic Signal Monitoring. Wuhan: Wuhan University of Technology, pp: 19-21.

[15]Kang Y H, Wu X J, Yang S Z. 2000. Magnetic non-destructive testing techniques in signal processing technology. NDT, 22(6): 58-61.

[16]Mou S, Chen Z P, Jin G L, Zhu N J. 2014. Advances in nondestructive testing technology rope. Mechatronics Engineering, 06: 707-710.

[17]Donglai Zhang, Min Zhao, Zhihui Zhou, Shimin Pan. 2013. Characterization of wire rope defects with gray level co-occurrence matrix of magnetic flux leakage images. Journal of Nondestructive Evaluation, 321.

[18]Esther-Sabrina Wacker, Joachim Denzler. 2013. Enhanced anomaly detection in wire ropes by combining structure and appearance. Pattern Recognition Letters, 348 . 


\section{MATEC Web of Conferences}

[19] Sheila Devasahayam, Veena Sahajwalla, Michael Sng. 2013. Investigation into failure in mining wire ropes-effect of crystallinity. Open Journal of Organic Polymer Materials, 0302.

[20]Pavel Peterka, Jozef Krešák, Stanislav Kropuch, Gabriel Fedorko, Vieroslav Molnar, Marek Vojtko. 2014. Failure analysis of hoisting steel wire rope. Engineering Failure Analysis, 45.

[21]Jishan Xia, Guohua Cao, Yandong Wang, Weihong Peng, A. S. Sekhar. 2014. Study on multicharacteristic of antirotation wire rope based on linear stiffness coefficient. Advances in Mechanical Engineering.

[22] Sung Ho Kim, Rjeon Hwan Bae, Jae Do Kwon. 2012. Bending fatigue characteristics of wire rope. Journal of Mechanical Science and Technology, 267.

[23]S. Moradi, K. Ranjbar, H. Makvandi. 2012. Failure analysis of a drilling wire rope. Journal of Failure Analysis and Prevention, 125.

[24] Hongyao Wang, Zhao Xu, Gang Hua, Jie Tian, Bingbing Zhou, Yanhong Lu, Fengjun Chen. 2009. Key technique of a detection sensor for coal mine wire ropes. Mining Science and Technology (China), 192.

[25]Corbett, Desi. 2009. Electric wire rope hoist for faster handling. FEN. 